\title{
Islam und Orient im Bild der Presse: Zur Suggestivkraft multimodaler Berichterstattung
}

Von Amr Abu Zeid, Marburg: Tectum Verlag 2016 (Zugl. Diss. Universität Bern 2014). ISBN 978-3-8288-3686-0. $260 \mathrm{~S}$

\section{Zouheir SOUKAH ${ }^{1 \oplus}$}

Dr., Himmelgeister Str. 72, 40225 Düsseldorf, Deutschland

Corresponding author:

Zouheir SOUKAH, Himmelgeister Str. 72, 40225 Düsseldorf, Deutschland

E-mail: zouheir.soukah@googlemail.com

Date of receipt: 06.05 .2018

Date of acceptance: 10.09 .2018

Citation: Soukah, Z. (2018). Islam und Orient im Bild der Presse: Zur Suggestivkraft multimodaler Berichterstattung [Review of the book Islam und Orient im Bild der Presse: Zur Suggestivkraft multimodaler Berichterstattung by A. A. Zeid]. Litera, 28(2), 263-268.

https://doi.org/10.26650/LITERA485767

Wir leben in einem ausgesprochenen Medienzeitalter. Die Wirkung der Massenmedien auf unserem öffentlichen sowie privaten Leben ist nun erheblich geworden. Mitverantwortlich sei hier auch der seit den letzten Jahren intensive Einsatz der Visualisierungen (wie Fotos, Bilder und Graphiken). Dies betont Amr Abu Zeid in seinem neu erschienen Buch Islam und Orient im Bild der Presse. Zur Suggestivkraft multimodaler Berichterstattung. Ein fast klassisch gewordenes Fallbeispiel dafür stellt zweifelsohne die Islamdarstellung in den Massenmedien - vor allem nach dem 11. September 2001 - dar, die seitdem immer mehr visualisiert wird. Durch ihre Visualisierungstechniken könnten sich Massenmedien zu den wichtigen Informationsvermittlern geworden, gerade über Islam und Muslime, also über Themen, zu denen die europäischen Rezipienten in der Regel keinen leichten Zugang 
haben. Aber wie gelang es den Massenmedien, eine führende Vermittlerrolle, gerade über ein akutes Thema wie den Islam und seine Wahrnehmung in der westlichen Öffentlichkeit, zu übernehmen. Genau dies versucht Amr Abu Zeid in Islam und Orient im Bild der Presse nachzugehen. Das Hauptverdient dieses Buches besteht allerdings vor allem darin, diesen immer wichtiger gewordenen islambezogenen Themen und deren Visualisierung mit Hilfe der modernen Bildsemiotik und der visuellen Bildrhetorik systematisch nachzugehen, denn Studien aus dem Bereich der Linguistik und insbesondere aus der Semiotik sind kaum zu finden. Abu Zaid nimmt als repräsentatives Korpus seiner Studie die zwei Nachrichtenmagazine Spiegel (Hamburg) und Weltwoche (Zürich) unter der Lupe und versucht, den textuellen sowie den visuellen Gehalt aller Ausgaben der beiden Wochenzeitschriften, die zwischen 2006 und 2010 erschienen, in Bezug auf Islamdarstellung systematisch zu untersuchen. Hier geht es ihm primär auf die drei folgenden Fragestellungen konkreter nachzugehen, nämlich:

- Wie können aus semiotischer Sicht visuelle Darstellungen die Rezipienten beeinflussen?

- Welche Darstellungsmechanismen werden in Massenmedien angewendet und vor allem zu welchem Zweck?

- Wie wird dabei die muslimische Minderheit in der deutschsprachigen Berichterstattung visuell wahrgenommen und bewertet?

Um diese Fragestellungen ausführlichen einzugehen, unterteilt der Autor sein Buch in neu eigenständigen Kapiteln, die meist aufeinander aufbauen. So fasst der Autor im ersten Kapitel (Einleitung) zunächst den Gesamtinhalt und die Zielsetzung seines Buches zusammen. Diese bestehe, so der Autor selbst, darin, „'sich stärker der visuellen Ebene in der Berichterstattung über islambezogene Themen [zu widmen]" (S. 15). Hierzu stellt er die in seiner Arbeit verwendeten Analysemethoden dar, nämlich die Verfahren der Bildsemiotik, die er für seine Untersuchung der IslamDarstellungen in den beiden Wochenzeitschriften Spiegel und Weltwoche anwendet. Folglich befasst sich das zweite Kapitel theoretisch mit der visuellen Kommunikation in der Presse im Allgemeinen. Im Zentrum dieses Kapitels stehen die Selektionsmechanismen, die eine sehr einschneidende Rolle bei der Auswahl von Bildern und Nachrichten spielen. Dabei zieht der Autor einen theoretischen Vergleich zwischen dem Medienbild und dem journalistischen Text gegenüber und kommt zu der Feststellung, dass das Bild im Zeitalter der Visualisierung einen eindeutigen 
Einfluss auf den Rezipienten ausübt:„Es veranschaulicht, informiert, erklärt, dekoriert, organisiert und strukturiert Zusammenhänge und kritisiert, unterhält, ruft Assoziationen hervor und stellt Verknüpfungen her" (S. 40). Hingegen findet der Leser im dritten Kapitel einen historischen Abriss über die markanten Auseinandersetzungen zwischen Europa und dem Islam seit seiner Entstehung. Dabei unterscheidet Abu Zaid zwischen den sogenannten ,bewaffneten' und den ,unbewaffneten' Begegnungen. Zu der ersten Kategorie gehören neben älteren Ereignissen wie Kreuzzügen und Türkenkriegen auch neuere Ereignisse, die noch einen spürbaren Einfluss auf unsere Gegenwart ausüben, wie der Kolonialismus, der arabisch-israelische Konflikt und nicht zuletzt der Krieg in Afghanistan. Zu den ,unbewaffneten' Begegnungen gehören, so der Autor, sowohl die gegenseitige Übersetzung als auch die Orientalistik. Dabei begründet der Autor die Integration dieses historischen Hintergrundes in sein Buch mit dem Ziel „,"ein besseres Verständnis des heutigen Islam- und Orientbildes" bei dem Leser zu ermöglichen.

Demzufolge setzt sich das vierte Kapitel statistisch mit der aktuellen Situation der muslimischen Minderheit in Europa und primär in Deutschland und der Schweiz auseinander. Zudem stellt er hier die islambezogenen Themen in Inlands- und Auslandsberichterstattung dar, die seit den letzten Jahren das mediale Islam- und Muslimbild maßgeblich gestaltet haben. Dazu gehören $u$. a. Themen wie ,Frauenrechte', ,Verschleierung', das ,Bauen von Moscheen', ,islamischer Extremismus', und nicht zuletzt,Terror'. Hier stellt Abu Zaid fest, dass diese Themenbereiche immer mehr visualisiert werden, und dass Visualisierungen sich mehr und mehr "als wichtiger Teil der medialen Nachricht neben dem sprachlichen Zeichen etablieren" (S. 97). Da Bilder auch die Thematik einer Nachricht mitformen, beschäftigt sich der Autor in den fünften und sechsten Kapiteln mit diesem wichtigen Aspekt der Bildsemiotik. Während sich das fünfte Kapitel auf Bild-Text- und Bild-Bild-Relationen anhand einiger konkreter Beispiele aus den beiden Nachrichtenmagazine beschränkt, entfaltet das sechste Kapitel weitere semiotische Aspekte vor allem aus der Kinesik, die Wissenschaft der Körperzeichen, deren drei Bestandteile, nämlich Mimik, Gestik und Körperhaltung „in der Medienkommunikation [...] wichtige Bedeutungsträger" (S. 109) bleiben. Diesbezüglich untersucht der Autor in diesem Kapitel ausgewählte Bilder aus den beiden Wochenzeitschriften, in denen immer wieder bestimmte Personen und Handlungen auftreten. Angrenzend werden auch die Aspekte der Gegenstände und Räume, die neben Personen und Handlungen auch eine wichtige Rolle in der Bildkommunikation spielen. Dies wird deutlich in der Analyse von den 
visuellen Moscheebildern. In diesem Rahmen kommt der Autor zu der Feststellung, dass die visuelle Darstellung der Moschee in den beiden zwei Wochenzeitschriften nicht nur die muslimischen Gebetshäuser als hinweisendes Zeichen auf das muslimische Andere, sondern meist als Fremdkörper, wenn sogar als Bedrohung bewertet (vgl., S. 148-167). Das siebte Kapitel widmet sich der Darstellung einiger rhetorischen Stilmittel, die bei der Gestaltung der Nachrichten immer mehr an Bedeutung gewinnen, da sie der Autor dazu anwendet, um „argumentative Zusammenhänge herzustellen sowie Überzeugungen zu erzeugen oder sie zu ändern" (S. 169). Zu diesen festgestellten rhetorischen Stilfiguren gehören: Anspielung, Analogien, Ellipsen sowie Antithesen und nicht zuletzt die Hyperbel. Dabei liefert der Autor konkrete Beispiele der Bildrhetorik, die sich mit islambezogenen Themen in den beiden Zeitschriften befassen und analysiert sie. Hier kommt er zu dem Ergebnis, dass sich der Einsatz dieser Stilfiguren nicht nur auf die visuelle Ebene beschränkt, sondern er betrifft vielmehr auch die Bildunterschriften sowie die Fließtexte selbst. Darüber hinaus beschäftigt sich der Autor im achten Kapitel mit der medialen Thematisierung und Visualisierung der Integration der muslimischen Minderheit sowohl in Deutschland als auch in der Schweiz. Hier diskutiert er, inwieweit Medien die muslimische Integration fördern können. Nach seiner Analyse einiger Darstellungsbeispiele aus der beiden Wochenzeitschriften bezüglich der Integrationsthematik kommt der Autor zu der Annahme, dass den Medien von der Seite der Muslime meist vorgeworfen wird, dass sie "durch eine tendenziöse Berichterstattung und durch eine undifferenzierte, kollektive Schuldzuweisung zur Verankerung eines negativen Bildes von ihnen im Bewusstsein des europäischen Rezipienten [beitrügen]" (S. 220). Und um nicht als ,Teil des Problems', sondern im Gegenteil als, Teil der Lösung' agieren zu können, schlägt der Autor den deutschsprachigen Medien drei mediale Lösungsansätze vor (vgl., S. 220223), damit sie "eine interkulturelle Funktion bei der Integration der muslimischen Minderheiten erfüllen und ihnen Akzeptanz verschaffen können“ (S. 220), obwohl dem Autor zufolge diese mediale Integration keinen Ersatz für andere wichtige Aspekte der Integration wie Sprache, Bildung und Arbeit darstellen kann. Folglich fast der Autor im neunten und somit letzten Kapitel (Schlussbetrachtung) die Ergebnisse seiner Arbeit zusammen. Dabei betont er zunächst, dass die untersuchten "Bilder in den meisten Fällen einen starken Einfluss auf die Gesamtaussage der journalistischen Nachricht ausüben" (S. 225). Ein weiteres wichtiges Ergebnis dieser Arbeit besagt, dass im untersuchten medialen Korpus meist "Bilder nicht-integrierter Muslime“ dominieren. Hingegen: „Integrierte Muslime werden als beispielhafte 
Einzelfälle präsentiert und sind oft weiblich“ (S. 231). Zum Schluss plädiert der Autor für wissenschaftliche Längsschnittuntersuchungen, die die verschiedenen islambezogenen Themen in der deutschsprachigen medialen Berichterstattung abdecken sollen. Dies betrachtet er als Notwendigkeit, um die Entwicklung sowie die Funktionen der medialen Visualisierung der islamischen Kultur im Westen besser zu verstehen. Mit diesem Plädoyer kann man nur einverstanden sein. Und zwar aus zwei wichtigen Gründen, nämlich: Wissenschaftliche Untersuchungen, die sich mit der medialen Islamdarstellung in den deutschsprachigen Raum befassen, sind bislang rar. Diese bilden bislang fast nur Kai Hafez The West and Islam in the mass media (2000) und Sabine Schiffers Die Darstellung des Islams in der Presse (2005). Dazu sind verstreute Artikel und Aufsätze in Sammelbänden vorzufinden, die sich jedoch selektiv mit diesem Thema befasst haben und somit nur begrenze Themenelemente der medialen Islamdarstellung abgedeckt haben. Der zweite Grund liegt darin, dass sich die einseitige Darstellung des Islams und die daraus entstandenen Negativurteile nicht nur in den Medien, sondern auch in der Öffentlichkeit in den letzten Jahren nur noch verschärft haben, was den Boden für Islamfeindlichkeit nährt. Leider wird diese potenzielle Relation zwischen der negativen Islamdarstellung in den Medien und die immer mehr zunehmende Islamfeindlichkeit in diesem Buch nicht untersucht. Darüber hinaus wurde dieser Aspekt bislang auch kaum genügend erforscht. Die einzige Ausnahme bilden die Arbeiten von Iman Attia, die sich aus soziowissenschaftlicher Hinsicht mit den Phänomenen Islamfeindlichkeit und antimuslimischer Rassismus befasst hat. Dazu gehören: Die ,westliche Kultur' und ihr Anderes. Zur Dekonstruktion von Orientalismus und antimuslimischem Rassismus (2009), und zuvor der Band Orient- und IslamBilder. Interdisziplinäre Beiträge zu Orientalismus und antimuslimischem Rassismus, den sie 2007 herausgegeben hat.

Anschließend es ist zu bemerken, dass die Überschrift des Buches Islam und Orient im Bild der Presse eindeutig suggeriert, dass dessen Hauptinteresse sowohl den Islam als auch den,Orient' und deren Medienbilder bilden. Dennoch beschränkt sich diese Untersuchung ausschließlich auf die mediale Darstellung des Islam und der Muslime im deutschsprachigen Raum. Ein weiterer wichtiger Aspekt, nämlich der ,Orient' als imaginative Vorstellung, nicht nur des muslimischen, fremden Anderen, sondern des europäischen deutschen Eigenen, wurde im Rahmen dieser Arbeit auch nicht berücksichtigt. Allerdings kann als Resümee festgestellt werden, dass dieses Buch eine eindeutige Lücke in der wissenschaftlichen Auseinandersetzung mit der medialen Wahrnehmung des Eigenen und des Anderen am Beispiel vom Islam und 
Muslimen auf einer immer wichtigeren Ebene, nämlich der der semiotischen bzw. rhetorischen Visualisierung abschließen konnte - in der Hoffnung, dass noch mehr empirische Untersuchungen aus anderen benachbarten Wissenschaften dieses komplexe Verhältnis zwischen dem Westen und dem Islam gerade auf der multimedialer Ebene systematisch untersuchen werden. 